WILLAM KONDO'

Adriane de Assis FISCHER De AstorI ${ }^{1}$

SURIA EL-Kouba Gomes'

Rachelle de BRIto FeRnandes ${ }^{1}$

MaRIa dAs GRaÇAS SASAKI ${ }^{2}$

Renato Luiz Sbalqueiro ${ }^{3}$

Artigos originais

Palavras-chave

Hepatopatias/induzido quimicamente Infecções por HIV/quimioterapia

HIV-1

Complicações infecciosas na gravidez Nevirapina/efeitos adversos

Nevirapina/toxicidade

Keywords

Liver diseases/chemically induced HIV infections/drug therapy

HIV-1

Pregnancy complications, infections Nevirapine/adverse effects Nevirapine/toxicity

Correspondência:

William Kondo

Avenida Getúlio Vargas, 3.163, apto. 21

CEP 80240-041 - Curitiba/PR

E-mail: williamkondo@yahoo.com

Recebido

03/01/2008

Aceito com modificacões

14/02/2008

\section{Avaliação dos efeitos colaterais da nevirapina em gestantes HIV positivo em Hospital Universitário do sul do Brasil}

\author{
Evaluation of the adverse effects of nevirapine in HIV-infected \\ pregnant women in a South Brazilian University Hospital
}

\section{Resumo}

OBJETIVO: avaliar a freqüência de efeitos advesos com o uso da nevirapina e suas correlações em gestantes infectadas pelo vírus da imunodeficiência humana (HIV). MÉTODOS: estudo retrospectivo foi realizado entre janeiro de 2003 e dezembro de 2006, incluindo todas as mulheres que utilizaram nevirapina durante a gestação. Os critérios de exclusão foram: início da nevirapina antes da gestação; presença de enzimas hepáticas basais aumentadas e dados incompletos de bioquímica hepática no prontuário. Os parâmetros avaliados foram idade, duração de exposição à nevirapina, idade gestacional no início da medicação, semanas de seguimento, carga viral, contagem de CD4 e dosagens de transaminases. A incidência de efeitos adversos hepáticos e/ou cutâneos foi determinada e correlacionada com a contagem de CD4. As análises estatísticas foram realizadas utilizando o teste exato de Fisher e o teste $t$ de Student quando apropriado. A significância estatística foi estabelecida quando $p \leq 0,05$. RESULTADOS: cento e cinqüenta e sete gestantes foram incluídas nos critérios estabelecidos. Trinta e uma mulheres (19,7\%) apresentaram toxicidade cutânea e/ou hepática. Rash cutâneo foi responsável por $77,4 \%$ das toxicidades e anormalidade da função hepática por 22,6\%. Hepatotoxicidades graus 1, 2 e 3 foram observadas em 0,6, 2,5 e 1,3\%, respectivamente. Contagem de CD4, carga viral e dosagem de transaminases basais foram similares em gestantes com e sem reação induzida pela nevirapina. A contagem de CD4 média foi de 465,4 e 416,6 células/ $\mu \mathrm{L}$ em mulheres com e sem efeitos colaterais, respectivamente $(p=0,3)$. Todas as pacientes que apresentaram hepatotoxicidade apresentavam contagem de CD4 prévia ao tratamento superior a 250 células/ $\mu$ L. CONCLUSÕES: a incidência de eventos adversos com nevirapina em nosso estudo foi alta, mas a maioria deles foi cutâneo. Não houve correlação entre a alta contagem de CD4 e os eventos adversos quando se analisou conjuntamente as reações cutâneas e hepáticas; entretanto, a hepatotoxicidade ocorreu apenas em gestantes com contagem de CD4 $\geq 250$ células/ $\mu \mathrm{L}$.

\section{Abstract}

PURPOSE: The aim of this article is to evaluate the use of nevirapine HIV-infected pregnant women in our service. METHODS: a retrospective study was performed between January 2003 and December 2006 analysing all women prescribed nevirapine in pregnancy. Exclusion criteria included: (1) women who started nevirapine before pregnancy, (2) patients with abnormal baseline liver enzymes, and (3) women with incomplete liver biochemistry data. Evaluated parameters included age, weeks of exposure to nevirapine, gestational age in the begginning of medication, weeks of follow-up, viral load, CD4 cells count and serum aminotransferase levels. The incidence of adverse hepatic and/or cutaneous effects was determined and correlated to the CD4 cells count. Statistical analysis were performed using Fisher's exact test and t-Student test when appropriate, with a statistical significance level of $p \leq 0,05$. RESULTS: one hundred fifty-seven women met the inclusion criteria. Thirtyone (19.7\%) presented cutaneous and/or hepatic toxicity. Skin rash accounted for $77.4 \%$ of toxicities and liver function abnormalities were noted in $22.6 \%$ of women exhibiting toxicities. Grade 1, 2 and 3 hepatotoxicities were observed in $0.6,2.5$ and $1.3 \%$, respectively. Baseline CD4 counts, viral loads and transaminases were similar in pregnant women with nevirapine adverse effects and those without reaction. Median absolute CD4 cell counts were 465.4 and 416.6 cells $/ \mu \mathrm{L}$ in women with and without side effects, respectively $(p=0.3)$. All patients who experienced hepatotoxicity had pretreatment CD4 counts superior to 250 cells/ $\mu \mathrm{L}$. CONCLUSIONS: The

Trabalho realizado no Departamento de Ginecologia e Obstetrícia do Hospital de Clínicas da Universidade Federal do Paraná UFPR - Curitiba (PR), Brasil.

'Residente de Ginecologia e Obstetrícia do Hospital de Clínicas da Universidade Federal do Paraná - UFPR - Curitiba (PR), Brasil. ${ }^{2}$ Infectologista do Hospital de Clínicas da Universidade Federal do Paraná - UFPR - Curitiba (PR), Brasil.

${ }^{3}$ Responsável pelo Pré-Natal de Alto Risco do Hospital de Clínicas da Universidade Federal do Paraná - UFPR - Curitiba (PR), Brasil. Conflito de interesses: Nenhum 
incidence of adverse events with nevirapine in our study was high, but most of them were cutaneous. There was no correlation between high CD4 counts and adverse events when analysing both cutaneous and hepatic reactions; nevertheless, hepatotoxicity occurred only in pregnant women with CD4 counts $\geq 250$ cells $/ \mu \mathrm{L}$.

\section{Introdução}

A terapia antiretroviral combinada é comumente prescrita para gestantes infectadas pelo vírus da imunodeficiência humana (HIV) para reduzir a carga viral materna e minimizar o risco de transmissão materno-fetal do HIV, assim como para o tratamento da doença materna quando indicado ${ }^{1-4}$. O objetivo do uso de antiretrovirais é o decréscimo da carga viral para menos que 1.000 cópias $/ \mathrm{mL}$ na época do parto ${ }^{4,5}$.

A nevirapina tem sido amplamente utilizada no Brasil como parte do esquema tríplice antiretroviral durante a gestação $0^{4,5}$. Trata-se de um potente inibidor da transcriptase reversa não nucleosídeo, bem absorvido por via oral e com distribuição transplacentária. Atinge concentração plasmática três horas após uma dose única de 200 mg e tem relativo baixo clearence, permitindo sua administração duas vezes ao dia. O perfil farmacocinético favorável da nevirapina permite dosagem simplificada e regime pouco oneroso para previnir a transmissão perinatal, útil especialmente em países em desenvolvimento ${ }^{6}$.

Recentemente, vários estudos têm levantado a questão da toxicidade da nevirapina administrada durante a gestação ${ }^{1,7}$. O rash é o efeito colateral cutâneo mais comum da nevirapina e sua incidência varia de acordo com os diferentes estudos ${ }^{8}$. Geralmente, aparece nas primeiras quatro semanas e pode resultar no desenvolvimento da síndrome de Stevens-Johnson ou necrólise epidérmica tóxica. A nevirapina também pode causar toxicidade hepática, variando desde hepatite assintomática até necrose hepática aguda e óbito ${ }^{7,9}$.

O intuito deste artigo é avaliar o uso da nevirapina em gestantes infectadas pelo HIV em serviço de atendimento de grávidas com sorologia positiva para HIV na região sul do Brasil.

\section{Métodos}

Foi realizado um estudo retrospectivo das gestantes com sorologia positiva que iniciaram o uso de nevirapina durante a gestação. As pacientes foram atendidas no PréNatal de Alto Risco durante o período de janeiro de 2003 a dezembro de 2006. O protocolo de estudo foi previamente aprovado pelo Comitê de Ética do Hospital das clínicas da Universidade Federal do Paraná (UFPR).

$\mathrm{O}$ grupo de estudo consistiu de gestantes infectadas pelo HIV tratadas com regime antiretroviral contendo nevirapina por um período superior a sete dias e que deram à luz com mais de 20 semanas de gestação. Não houve restrição com relação à contagem de CD4 para inclusão no estudo. Os critérios de exclusão foram: mulheres que iniciaram a nevirapina antes da gestação; pacientes com enzimas hepáticas aumentadas na dosagem basal (ALT superior a 2,5 vezes o limite superior da normalidade) e mulheres com dados incompletos de bioquímica hepática (menos do que uma dosagem pré-tratamento e duas dosagens durante o uso da medicação).

Todas as pacientes incluídas no estudo tiveram o diagnóstico de infecção pelo HIV confirmado de acordo com o protocolo do Ministério da Saúde ${ }^{10}$ : triagem sorológica com imunoensaio (Enzyme-Linked ImmunoSorbent Assay, ELISA); em caso de amostra com resultado reagente ou inconclusivo nesse primeiro imunoensaio, confirmação sorológica com um segundo imunoensaio (diferente do primeiro na sua constituição antigênica ou princípio metodológico) e testes confirmatórios, tais como a imunofluorescência indireta, imunoblot ou western blot. As pacientes que já tinham o diagnóstico confirmado de infecção pelo HIV e já utilizavam medicação antiretroviral só foram incluídas no estudo caso nunca tivessem feito uso de nevirapina.

A nevirapina foi sempre prescrita associada à zidovudina e à lamivudina, em uma dosagem de $200 \mathrm{mg}$ ao dia durante os primeiros 14 dias. $\mathrm{Na}$ ausência de qualquer manifestação cutânea após essas duas semanas iniciais, dose plena (200 mg duas vezes ao dia) foi instituída .

Os dados foram coletados por meio da revisão dos prontuários, incluindo história clínica e dados demográficos e sociais (incluindo uso de álcool e drogas ilícitas). História clínica, exame físico, uso concomitante de outras medicações, efeitos colaterais (rash cutâneo, prurido, icterícia, colúria, acolia etc.) e necessidade de substituição do esquema antiretroviral foram revisados. Os dados laboratoriais pesquisados incluíram hemograma completo, dosagem de alanina aminotransferase (ALT) e aspartato aminotransferase (AST) basal e no seguimento, sorologias para hepatite B e C, contagem de CD4 e carga viral. Todas as gestantes com contagem de CD4 inferior a 200 células/ $\mu$ l utilizaram profilaxia com sulfametoxazol-trimetoprim.

Durante o período, 180 mulheres foram identificadas como usuárias de nevirapina como parte da terapia antiretroviral combinada durante a gestação, das quais 157 foram eleitas para análise. As 23 gestantes restantes foram excluídas, pois dez já estavam utilizando nevirapina antes da gestação e 13 apresentavam dados incompletos de prontuário. 
A incidência de toxicidade cutânea e/ou hepática foi estimada de acordo com a escala de graduação de toxicidade do National Institute of Allergy and Infectious Diseases Division of AIDS. A severidade dos níveis de AST e ALT foram graduadas de acordo com a classificação de toxicidade do National Institute of Allergy and Infectious Diseases ${ }^{1}$ : grau $0 \leq 1,25$ vezes o limite superior da normalidade; grau $1=1,25$ 2,5 vezes o limite superior da normalidade (hepatotoxicidade leve); grau $2=2,5-5,0$ vezes o limite superior da normalidade (hepatotoxicidade leve); grau 3=5,0-10,0 vezes o limite superior da normalidade (hepatotoxicidade severa); grau $4 \geq 10$ vezes o limite superior da normalidade (hepatotoxicidade severa).

As pacientes que apresentaram efeitos colaterais induzidos pela nevirapina foram estratificadas de acordo com a contagem de CD4 e separadas em dois grupos (menor ou igual a 250 células/ $\mu \mathrm{L}$ e maior do que 250 células/ $\mu \mathrm{L}$ ) para tentar correlacionar a ocorrência de efeitos colaterais e a contagem de CD4. Na presença de efeitos colaterais, sempre foi optado por alterar o esquema antiretroviral.

As análises estatísticas foram realizadas utilizando o programa STATISTICA 5.5 utilizando o teste exato de Fisher e o teste $t$ de Student. O teste $t$ de Student foi utilizado para comparar as médias das variáveis independentes entre os grupos com e sem efeitos colaterais (idade, duração de exposição à nevirapina, idade gestacional no início da medicação, semanas de seguimento, carga viral, contagem de CD4 e dosagens de transaminases). O teste exato de Fisher foi utilizado para avaliar a associação entre a contagem de CD4 ( $\leq$ ou $>250$ células $/ \mu \mathrm{L}$ ) e a presença ou não de efeitos colaterais. A significância estatística foi estabelecida quando $\mathrm{p} \leq 0,05$.

\section{Resultados}

A média de idade das gestantes foi de 27,9 96,2 anos e o diagnóstico da infecção pelo HIV foi realizado durante a gestação em $49 \%$ dos casos. Com relação aos fatores de risco para hepatotoxicidade, seis gestantes eram portadoras de hepatite $\mathrm{B}$, sete eram portadoras de hepatite $\mathrm{C}$ e dez eram usuárias de drogas injetáveis.

Dentre as gestantes incluídas no estudo, $31(19,7 \%)$ apresentaram toxicidade cutânea e/ou hepática com o uso da nevirapina. As características clínicas e laboratoriais de base das pacientes estão descritas na Tabela 1 .

A terapia antiretroviral contendo nevirapina foi instituída em média na $24^{a}$ semana de gestação, sendo na $22^{\mathrm{a}}$ semana nas pacientes com efeitos colaterais e na $24^{a}$ semana naquelas sem efeitos colaterais $(p=0,25)$.

A contagem de $\mathrm{CD} 4$, a carga viral e as transaminases de base estavam disponíveis em todas as pacientes analisadas e não houve diferença significativa nessas características basais entre as mulheres com e sem EF. A contagem média de CD4 foi de 465,4 e 416,6 células/ $\mu \mathrm{L}$ nas mulheres com e sem EF, respectivamente, sem diferença significativa $(\mathrm{p}=0,3)$. O tempo de exposição à nevirapina variou de 7 a 189 dias, média de 26,6 dias nas mulheres com EF e 101,5 dias nas pacientes sem $\operatorname{EF}(p<0,01)$. Essa diferença ocorreu por causa da suspensão da nevirapina no grupo que apresentou efeitos adversos.

Correlacionando a contagem de CD4 e a presença de efeitos colaterais, observamos que quatro de 37 mulheres com contagem de CD4 <250 células/ $\mu \mathrm{L}(10,8 \%)$ e 27 de 120 mulheres com contagem de CD $4 \geq 250$ células/ $\mu \mathrm{L}(22,5 \%)$ desenvolveram efeitos adversos, sem diferença significativa entre os grupos $(\mathrm{p}=0,11)$.

O tempo de início dos efeitos colaterais variou de 7 a 49 dias (média de 25,2 $\pm 12,2$ dias) após a instituição do tratamento com nevirapina. O rash cutâneo correspondeu a $77,4 \%$ de todos os casos de toxicidade ( 24 de 31 mulheres) e a $15,3 \%$ de todas as pacientes recebendo nevirapina. As alterações da função hepática foram notadas em $22,6 \%$ das mulheres que exibiram toxicidade ( 7 de 31 mulheres) e em $4,5 \%$ do número total de pacientes recebendo nevirapina. Nenhuma gestante apresentou rash cutâneo e alteração hepática simultaneamente (Tabela 2). Hepatotoxicidade grau 1, 2 e 3 foi observada em uma, quatro e duas gestantes, respectivamente. As pacientes com hepatotoxidades grau 2 e 3

Tabela 1 - Características clínicas e laboratoriais de 157 gestantes infectadas pelo HIV usando nevirapina segundo a presença ou ausência de efeitos colaterais (EF).

\begin{tabular}{|c|c|c|c|c|}
\hline & $\begin{array}{c}\text { EF } \\
(n=31)\end{array}$ & $\begin{array}{c}\text { Ausência de EF } \\
(n=126)\end{array}$ & $\begin{array}{c}\text { Total } \\
(n=157)\end{array}$ & $\begin{array}{l}\text { Valor } \\
\text { de } p\end{array}$ \\
\hline Idade (anos) & $30 \pm 6,2$ & $27,4 \pm 6,1$ & $27,9 \pm 6,2(14-43)$ & 0,03 \\
\hline $\begin{array}{l}\text { Duração da } \\
\text { exposiçōo à } \\
\text { nevirapina (semanas) }\end{array}$ & $3,8 \pm 1,8$ & $14,5 \pm 6,8$ & $12,3 \pm 7,5(1-27)$ & $<0,01$ \\
\hline $\begin{array}{l}\text { Semanas de } \\
\text { gestação no início } \\
\text { da medicação }\end{array}$ & $22,7 \pm 7,8$ & $24,5 \pm 7$ & $24,1 \pm 7,2(10-37)$ & 0,25 \\
\hline $\begin{array}{l}\text { Semanas de } \\
\text { seguimento }\end{array}$ & $15,8 \pm 7,9$ & $14,5 \pm 6,8$ & $14,8 \pm 7(1-27)$ & 0,38 \\
\hline $\begin{array}{l}\text { Carga viral" } \\
\text { (cópias/mL) }\end{array}$ & $20980,3 \pm 32169$ & $28541,5 \pm 76107,8$ & $\begin{array}{l}\text { 27098,9 } 969862,2 \\
\text { (indetectável- } \\
730000 \text { ) }\end{array}$ & 0,41 \\
\hline $\begin{array}{l}\text { Contagem de CD4* } \\
\text { (células } / \mu \mathrm{L} \text { ) }\end{array}$ & $465,4 \pm 218,9$ & $416,6 \pm 227,1$ & $\begin{array}{c}425,8 \pm 225,7 \\
(43-1218)\end{array}$ & 0,3 \\
\hline AST* $^{*}(\mathrm{U} / \mathrm{L})$ & $20,2 \pm 6,7$ & $20,9 \pm 7,4$ & $20,8 \pm 7,3(7-52)$ & 0,73 \\
\hline ALT* (U/L) $^{*}$ & $16,4 \pm 9$ & $17,4 \pm 8,1$ & $17,2 \pm 8,2(5-54)$ & 0,67 \\
\hline
\end{tabular}

Nota: todos os parâmetros são apresentados como: média em cada subgrupo \pm desvio padrão (valor mínimo-valor máximo). "Valores prévios ao início da medicação.

Tabela 2 - Eventos adversos em mulheres utilizando terapia com nevirapina.

\begin{tabular}{l|ccc}
\hline & CD4 $<250(n=37)$ & $C D 4 \geq 250(n=120)$ & Total $(\mathrm{n}=157)$ \\
\hline Rash & $4(10,8 \%)$ & $20(16,7 \%)$ & $24(15,3 \%)$ \\
Hepatofoxicidade & & & \\
Grau 1 & & $1(0,8 \%)$ & $1(0,65 \%)$ \\
Grau 2 & - & $4(3,3 \%)$ & $4(2,55 \%)$ \\
Grau 3 & - & $2(1,7 \%)$ & $2(1,3 \%)$ \\
Grau 4 & - & - & - \\
\hline
\end{tabular}


foram internadas e, após a interrupção da nevirapina, todas apresentaram melhorara clínica e laboratorial.

O tempo de seguimento das gestantes com e sem EF desde o início do pré-natal até o parto foi de 15,8 e 14,5 semanas, respectivamente $(\mathrm{p}=0,38)$.

\section{Discussão}

A eficácia da terapia antiretroviral para prevenir a transmissão materno-fetal do HIV foi estabelecida pela primeira vez em 1994. Neste ano, Connor et al. (PACTG 076) ${ }^{11}$ observaram redução da transmissão do HIV de 25 para $8,5 \%$ quando tanto a mãe quanto o recém-nascido foram tratados com zidovudina nos períodos pré-natal, intraparto e pós-parto. Subseqüentemente, vários autores demonstraram que a monoterapia com zidovudina tinha pequeno efeito na carga viral materna ${ }^{12}$ e podia acarretar a transmissão de formas mutantes do HIV, resistentes à zidovudina ${ }^{13}$. O estudo PETRA foi designado para tentar verificar se a terapia combinada (zidovudina e lamivudina) poderia ter benefício adicional àquele oferecido pela monoterapia com zidovudina em decorrência de uma provável diminuição da carga viral materna. A redução significativa da transmissão perinatal do HIV com o uso da terapia combinada foi observada, mas os dados referentes à queda da carga viral materna não foram apresentados ${ }^{14}$.

Como apenas uma mutação (M184V) no gene da transcriptase reversa do HIV é necessária para causar grande resistência à lamivudina, a combinação de zidovudina e lamivudina pode não ser suficiente para manter supressão viral em alguns indivíduos ${ }^{15}$. O rápido desenvolvimento de resistência à lamivudina em até $80 \%$ dos vírus foi descrito com o uso dessa combinação durante a gestação ${ }^{13}$. Uma supressão viral mais completa e duradoura pode ser possível com o uso de HAART (highly active antiretroviral therapy, ou seja, terapia antiretroviral altamente ativa), composta de inibidores da transcriptase reversa nucleosídeos (como a zidovudina e a lamivudina) associados a um inibidor da transcriptase reversa não nucleosídeo (como a nevirapina) e/ou um inibidor da protease (como o nelfinavir). Com esse esquema terapêutico, vários estudos retrospectivos têm relatado redução nas taxas de transmissão perinatal do HIV para menos de $2 \%$.

De acordo com o guidelines brasileiros de 2006 para o manejo da infecção pelo HIV na gestação, todas as mulheres grávidas infectadas pelo HIV devem utilizar durante o pré-natal regime antiretroviral com zidovudina isolada ou com esquema tríplice, geralmente incluindo nevirapina ou nelfinavir, dependendo da contagem de CD4 e da carga viral. A escolha entre o nelfinavir e a nevirapina é baseada nas semanas de gestação, estado imune, carga viral e aderência ao tratamento e ao seguimento clínico ${ }^{5}$. Após o aviso de recolhimento do nelfinavir pelo laboratório Roche em junho de 2007, a associação lopinavir/ritonavir passou a substituir o nelfinavir durante a gestação. No entanto, nossa amostra abrangeu apenas pacientes tratadas antes dessa data.

Estudos têm demonstrado que os regimes de HAART contendo um inibidor de transcriptase reversa não nucleosídeo são efetivos e bem tolerados, mas efeitos adversos como reação de hipersensibilidade e hepatotoxicidade são relativamente comuns ${ }^{16}$.

Altas taxas de rash cutâneo associado à nevirapina foram relatadas em pacientes da Tailândia e China ${ }^{17,18}$, com uma associação entre altas contagens de $\mathrm{CD} 4$ e risco de rash cutâneo severo associado à droga ${ }^{8}$. Rash severo ocorre em aproximadamente $2 \%$ das pacientes recebendo nevirapina.

Aumentos assintomáticos nos níveis de AST ou ALT de mais de cinco vezes o limite superior do normal ocorrem em cerca de $9 \%$ das pacientes utilizando nevirapina (comparado a 6\% dos controles). Hepatite sintomática é observada em cerca de $4 \%$ das pacientes tratadas com a droga. Os fatores que têm sido relacionados à toxididade da nevirapina incluem: tempo de exposição à nevirapina ${ }^{9}$, altas contagens de CD4 ( $\geq 250$ células $/ \mu \mathrm{L})^{1,7,10,19}$, coinfecção por hepatite $\mathrm{B}$ ou $\mathrm{C}^{9,10,20}$, elevação das enzimas hepáticas de base ${ }^{19}$ e abuso de álcool e drogas ${ }^{9,20}$. Em estudos clínicos controlados, eventos clínicos hepáticos, independente da severidade, ocorreram em $4 \%$ (variando de 2,5 a $11 \%$ ) das pacientes que receberam nevirapina; no entanto, o risco de falência hepática associada à nevirapina ou mortalidade de causa hepática tem sido baixo, variando entre 0,04 e $0,4 \%{ }^{19}$. O estudo recente de Jamisse et al. ${ }^{21}$ demonstrou que a hepatotoxicidade severa foi mais comum com altas contagens de CD4 (6\% vs. $0 \%$ entre mulheres com contagens de CD $4 \geq 250$ células $/ \mu \mathrm{L}$ e $<250$ células $/ \mu \mathrm{L}$, respectivamente). Entretanto, na coorte de João et al. ${ }^{4}$, apenas as contagens de CD4 superiores a 600 células/ $\mu \mathrm{L}$ demonstraram aumento significativo do risco para toxicidade.

Hepatotoxicidade severa e em alguns casos fatal - incluindo hepatite fulminante e colestática, necrose hepática e falência hepática, e reações de hipersensibilidade cutânea graves, incluindo síndrome de Stevens-Johnson - têm sido relatadas em pacientes infectados pelo HIV que recebem nevirapina em combinação com outras drogas para o tratamento do HIV e em um pequeno número de indivíduos que recebem nevirapina como parte do regime para a profilaxia pós-exposição nosocomial ou sexual do HIV. Estas toxicidades também têm sido relatadas em mulheres grávidas infectadas pelo HIV utilizando tratamento antiretroviral contendo nevirapina para a prevenção da transmissão perinatal ${ }^{2-4,21,22}$. O maior risco para rash severo ou eventos hepáticos ocorre durante as primeiras seis semanas de terapia ${ }^{10,22}$, embora o risco de toxicidade continue após esse período e a monitorização deva continuar em intervalos freqüentes. Falência hepática pode progredir em alguns casos, apesar da interrupção da droga ${ }^{22}$. 
Até hoje, foram relatados pelo menos oito casos de hepatite fulminante culminando em morte materna relacionados à terapia antiretroviral contendo nevirapina na gestação. Os achados em comum de seis casos relatados em uma das séries incluíram falência hepática fulminante se apresentando quatro a cinco semanas após o início da terapia, em mulheres virgens de tratamento, com dosagem prévia de ALT normal e sem história de hepatite $\mathrm{B}$ ou $\mathrm{C}^{1}$. Todos os casos ocorreram em mulheres com contagem de CD4 de base superior a 250 células $/ \mu \mathrm{L}$. Além disso, nesses casos, a hepatite progrediu rapidamente para falência hepática, apesar da interrupção da nevirapina e da monitorização clínica e laboratorial intensiva.

A incidência de efeitos adversos em nossa série de gestantes utilizando nevirapina foi de $19,7 \%$. O tempo para o desenvolvimento dos efeitos adversos foi de 7 a 49 dias, com média de 25 dias. Todos os efeitos adversos da nevirapina se desenvolveram em menos de sete semanas, como observado em outras coortes de mulheres grávidas ${ }^{1,4,21}$. Embora a toxicidade grau 3 tenha sido detectada em 1,3\% (dois casos) das gestantes, as elevações das transaminases não resultaram em hepatotoxicidade clinicamente significativa. Também observamos $2,5 \%$ (quatro casos) e $0,6 \%$ (um caso) de hepatotoxicidade graus 2 e 1 , respectivamente. As pacientes com hepatotoxicidades grau 2 e 3 foram hospitalizadas e todos os casos se trataram de elevações transitórias das transaminases, que regrediram após a interrupção da nevirapina. Todos os casos de elevação das enzimas hepáticas ocorreram em mulheres com contagem de CD4 igual ou superior a 250 células $/ \mu \mathrm{L}$ em nosso estudo. As 24 pacientes restantes $(15,3 \%)$ desenvolveram rash cutâneo com ou sem prurido, com regressão espontânea após a suspensão da medicação.

A contagem $\mathrm{CD} 4$ e a carga viral basais não diferiram significativamente entre nossas pacientes com e sem efeitos adversos. As reações foram observadas em 22,5 e $10,8 \%$ das pacientes com contagem de CD $4 \geq 250$ células $/ \mu \mathrm{L}$ $\mathrm{e}<250$ células/ $\mu \mathrm{L}$, respectivamente, mas esta diferença não atingiu significância $(\mathrm{p}=0,1)$. Kramer et al. ${ }^{23}$ também não encontraram diferença significativa na contagem de CD4 em gestantes com e sem toxicidade da nevirapina. Uma publicação recente na qual foram avaliadas 235 gestantes infectadas pelo HIV utilizando terapia antiretroviral contendo nevirapina concluiu que a medicação é bem tolerada e que a contagem de CD4 pode ser menos preditiva da ocorrência de toxicidade na gestação ${ }^{24}$. Achados similares já haviam sido publicados anteriormente em estudo com gestantes africanas usando terapia antiretroviral contendo nevirapina ${ }^{25}$.

É interessante que nossos dados não confirmaram a associação entre altas contagens de CD4 e os efeitos adversos induzidos pela nevirapina, quando consideramos tanto as reações cutâneas quanto as hepáticas. No entanto, quando consideramos apenas a hepatotoxicidade, observamos que todos os casos de alterações nas enzimas hepáticas ocorreram em pacientes com contagem de $\mathrm{CD} 4 \geq 250$ células $/ \mu \mathrm{L}$, confirmando a correlação entre altas contagens de CD4 e a toxicidade hepática. Esta observação é compatível com a hipótese de que a hepatotoxicidade seja causada por efeito tóxico imunomediado no fígado, que é mais rápido quando o sistema imune está menos comprometido.

Ainda não se sabe se a gestação realmente aumenta o risco de hepatotoxicidade em mulheres utilizando nevirapina ou outras drogas antiretrovirais. Como a gravidez pode por si só mimetizar alguns dos sintomas precoces da hepatotoxicidade (colestase intra-hepática, fígado gorduroso, pré-eclâmpsia e eclâmpsia), os profissionais da área de saúde que prestam atendimento a gestantes utilizando nevirapina devem estar atentos a essa potencial complicação e realizar monitorização freqüente e cuidadosa dos sinais e sintomas clínicos e das transaminases, particularmente durante as primeiras 18 semanas de terapia 5 .

Em resumo, observamos alta incidência de efeitos colaterais relacionados à terapia antiretroviral contendo nevirapina em nossas pacientes, sendo a maioria rash cutâneo sem elevação de transaminases. Não houve correlação entre contagem de $\mathrm{CD} 4$ e efeitos colaterais induzidos pela nevirapina quando tanto os eventos cutâneos quanto os hepáticos foram considerados; entretanto, todos os casos de toxicidade hepática ocorreram em pacientes com contagem de CD $4 \geq 250$ células $/ \mathrm{mL}$. Embasados nesses resultados, passamos a utilizar a nevirapina apenas nas gestantes portadoras do HIV com CD $4<250$ células/mL ou naquelas com CD4 $\geq 250$ células/mL, que apresentaram efeitos colaterais com outras drogas.

\section{Referências}

1. Hitti J, Frenkel LM, Stek AM, Nachman SA, Baker D, GonzalezGarcia A, et al. Maternal toxicity with continuous nevirapine in pregnancy: results from PACTG 1022. J Acquir Immune Defic Syndr. 2004;36(3):772-6.
2. Lyons F, Hopkins S, Kelleher B, McGeary A, Sheehan G, Geoghegan J, et al. Maternal hepatotoxicity with nevirapine as part of combination antiretroviral therapy in pregnancy. HIV Med. 2006;7(4):255-60. 
3. Joy $S$, Poi $M$, Hughes $L$, Brady MT, Koletar SL, Para MF, et al. Third-trimester maternal toxicity with nevirapine use in pregnancy. Obstet Gynecol. 2005; 106 (5 Pt 1):1032-8.

4. João EC, Calvet GA, Menezes JA, D'Ippolito MM, Cruz ML, Salgado LA, et al. Nevirapine toxicity in a cohort of HIV-1-infected pregnant women. Am J Obstet Gynecol. 2006;194(1):199-202.

5. Brasil - Ministério da Saúde. Recomendações para profilaxia da transmissão vertical do HIV e terapia anti-retroviral em gestantes [documento da Internet]. Brasília: 2006 [citado 18 dez 2007]. Disponível em: http://www.saude.rio.ri.gov.br/media/dstaids_ consenso_gestante_2006.pdf

6. Guay LA, Musoke P, Fleming T, Bagenda D, Allen M, Nakabiito C, et al. Intrapartum and neonatal single-dose nevirapine compared with zidovudine for prevention of mother-to-child transmission of HIV-1 in Kampala, Uganda: HIVNET 012 randomised trial. Lancet. 1999;354(9181):795-802.

7. de Maat MM, ter Heine R, van Gorp EC, Mulder JW, Mairuhu AT, Beijnen JH. Case series of acute hepatitis in a non-selected group of HIV-infected patients on nevirapine-containing antiretroviral treatment. AIDS. 2003;17(15):2209-14.

8. Bersoff-Matcha SJ, Miller WC, Aberg JA, van Der Horst C, Hamrick HJ Jr, Powderly WG, et al. Sex differences in nevirapine rash. Clin Infect Dis. 2001;32(1):124-9.

9. Martín-Carbonero L, Núñez M, González-Lahoz J, Soriano V. Incidence of liver injury after beginning antiretroviral therapy with efavirenz or nevirapine. HIV Clin Trials. 2003;4(2):1 15-20.

10. Brasil - Ministério da Saúde [documento da Internet]. Protocolo para a prevenção de transmissão vertical de HIV e sífilis. Brasília: 2006 [citado 18 dez 2007]. Disponível em: http://www.saude. rio.ri.gov.br/media/dstaids_gestante_protocolo_out06.pdf

11. Connor EM, Sperling RS, Gelber R, Kiselev P, Scott G, O'Sullivan $M$, et al. Reduction of maternal-infant transmission of human immunodeficiency virus type 1 with zidovudine treatment. Pediatric AIDS Clinical Trials Group Protocol 076 Study Group. N Engl J Med. 1994;331(18):1173-80.

12. Garcia PM, Kalish LA, Pitt J, Minkoff H, Quinn TC, Burchett SK, et al. Maternal levels of plasma human immunodeficiency virus type 1 RNA and the risk of perinatal transmission. Women and Infants Transmission Study Group. N Engl J Med. 1999;341(6):394-402.

13. Clarke JR, Braganza R, Mirza A, Stainsby C, Ait-Khaled M, Wright $A$, et al. Rapid development of genotypic resistance to lamivudine when combined with zidovudine in pregnancy. J Med Virol. 1999;59(3):364-8
14. Saba J. The results of the PETRA intervention trial to prevent perinatal transmission in sub-Saharan Africa. Abstract of the 6th Conference on Retroviruses and Opportunistic Infections; 1999 Jan 31-Feb 4; Chicago, USA. Alexandria: Foundation for Retrovirology and Human Health; 1999. p. S6.

15. McGowan JP, Shah SS. Management of HIV infection during pregnancy. Curr Opin Obstet Gynecol. 2000;12(5):357-67.

16. Carr A, Cooper DA. Adverse effects of antiretroviral therapy. Lancet. 2000;356(9239): 1423-30.

17. Ananworanich J, Moor Z, Siangphoe U, Chan J, Cardiello P, Duncombe $C$, et al. Incidence and risk factors for rash in Thai patients randomized to regimens with nevirapine, efavirenz or both drugs. AIDS. 2005;19(2):185-92.

18. Ho TT, Wong KH, Chan KC, Lee SS. High incidence of nevirapine-associated rash in HIV-infected Chinese. AIDS. 1998; 12(15):2082-3.

19. Stern JO, Robinson PA, Love J, Lanes S, Imperiale MS, Mayers DL. A comprehensive hepatic safety analysis of nevirapine in different populations of HIV infected patients. J Acquir Immune Defic Syndr. 2003;34 Suppl 1:S21-33.

20. Núñez M, Lana R, Mendoza JL, Martín-Carbonero L, Soriano $V$. Risk factors for severe hepatic injury after introduction of highly active antiretroviral therapy. J Acquir Immune Defic Syndr. $2001 ; 27(5): 426-31$

21. Jamisse L, Balkus J, Hitti J, Gloyd S, Manuel R, Osman N, et al. Antiretroviral-associated toxicity among HIV-1-seropositive pregnant women in Mozambique receiving nevirapine-based regimens. J Acquir Immune Defic Syndr. 2007;44(4):371-6.

22. Wooltorton E. HIV drug nevirapine (Viramune): risk of severe hepatotoxicity. CMAJ. 2004;170(7):1091.

23. Kramer F, Stek A, Du WB, Kovacs A. Nevirapine tolerability in HIVinfected women in pregnancy. Abstract of the 11 th Conference on Retrovirus and Opportunistic Infections; Feb 2004; San Francisco, USA. Alexandria: Foundation for Retrovirology and Human Health; 2004. p. 923.

24. Natarajan U, Pym A, McDonald C, Velisetty P, Edwards SG, Hay $P$, et al. Safety of nevirapine in pregnancy. HIV Med. 2007;8(1):64-9.

25. Marazzi MC, Germano P, Liotta G, Guidotti G, Loureiro S, da Cruz Gomes A, et al. Safety of nevirapine-containing antiretroviral triple therapy regimens to prevent vertical transmission in an African cohort of HIV-1-infected pregnant women. HIV Med. 2006;7(5):338-44. 